\title{
Shape memory polyurethanes reinforced with carbon nanotubes
}

\author{
M.A. Fonseca ${ }^{\text {a,* }}$, B. Abreu ${ }^{\text {a }}$, F.A.M.M. Gonçalves ${ }^{\text {b }}$, A.G.M. Ferreira ${ }^{\text {b }}$, R.A.S. Moreira ${ }^{\text {a }}$, M.S.A. Oliveira ${ }^{\text {a }}$ \\ ${ }^{a}$ Department of Mechanical Engineering, University of Aveiro, 3810-193 Aveiro, Portugal \\ ${ }^{\mathrm{b}}$ Department of Chemical Engineering, University of Coimbra, Pólo II, Rua Silvio Lima, 3030-790 Coimbra, Portugal
}

\section{A R T I C L E I N F O}

Article history:

Available online 20 December 2012

\section{Keywords:}

Composites

SMPU/CNT

In situ polymerization

Melt mixing

\begin{abstract}
A B S T R A C T
New shape-memory materials (SMMs) for applications in active control and morphing structures have been attracting special attention due to its unique properties. These SMM can be metallic alloys (SMAs), piezoelectric, and polymers such as polyurethanes (SMPUs). The latter detain higher recovery rates but better processability, however, the reaction time is longer when compared with the SMA.

The addition of carbon nanotubes (CNTs) to SMPU seems to improve its overall properties with a great deal of potential in what concerns improved shape memory. There are two main techniques to attain SMPU/CNts nanocomposites: in situ polymerization and mechanical melt mixing. The study here presented establishes a comparison between these two techniques. To assess the suitability of the latter a rather extensive characterization was carried out. The homogeneity of the CNTs dispersion into the polymer matrix was established through SEM and the thermal characterization has shown a rise in the glass transition temperature consistent with CNT loading. Furthermore, shape memory seems to improve with the nanoparticle reinforcement. Within the two processing techniques it could be referred that melt processing seems to be simpler to use with better laboratory repeatability, thus detaining a greater potential should nanocomposite tailoring at a larger scale be envisaged.
\end{abstract}

() 2012 Elsevier Ltd. All rights reserved.

\section{Introduction}

In the last few years, some efforts have been taken towards the introduction of control mechanisms to enable a structure to adapt itself when facing variable operation conditions or when a set of specialized features, which improve the structure performance to attain specific goals, are required. Certain structures require a continuous and controlled shape reconfiguration or motion control during its operation in order to react against external and variable actions, like vibrations and noise, usually denoted as active control [1]. Others rely on its ability to continuously change its configuration, following a pre-determined set of intended features or as a reaction to the action of external variables. This ability is usually known as shape control [2-4]. Finally, some structures are designed to be used under extreme and contrary limits where the complete reconfiguration of its shape is a mandatory requirement to be well succeed in its mission. This feature usually mimics the capacity of living structures to change radically its shape, dimension or surface characteristics (color, reflectivity, roughness) and is usually described as morphing capability. Morphing has becoming one of the major research lines in the aeronautic, aerospace and marine structural fields [1].

\footnotetext{
* Corresponding author. Tel.: +351 234370 830; fax: +351 234370953.

E-mail address: mfonseca@ua.pt (M.A. Fonseca).
}

Piezoelectric (PZT) materials and shape memory alloys (SMAs) are some examples of the currently applied solutions for active control [5,6], shape control [2-4] and morphing [7,8]. PZT patches are the most investigated and applied active material, especially for active and shape control, whereas SMAs activated by a thermal trigger were considered as active fibers embedded within structural materials [9] for distributed actuation mechanisms. Despite the relative success of PZT and SMA materials as solutions for active control, due to its inherent fast response capacity, when large and distributed deformations are required, as those demanded by shape control of medium/large structures and morphing approaches, the discrete use of these materials present some drawbacks, often requiring the use of distribution and amplifying mechanism to transform a localized, small and usually unidirectional actuation into a large, global and multidirectional deformation patterns required to attain the target shape.

Recently, a new class of shape memory materials has gained a special attention in this research field, the shape memory polymers (SMPs), which can be deformed and maintained at a transitory shape until receiving a special stimulus, or trigger, that forces the recovering of the initial and permanent configuration. One of the most important classes of SMPs are the polyurethanes. This class of polymers is formed by soft and hard segments. The hard segments binding each other through interactions as hydrogen bonding, dipole-dipole interaction, and Van der Waals forces, work as physical crosslinking points and in some way restore the original 
shape after deformation. On the other hand, soft/flexible segments absorb external stress during elongation or compression and determine the phase-transition temperature.

SMPs have some advantages when compared to the SMAs and PZTs: SMP materials detain higher elastic deformations (strain up to $200 \%$ ), present a lower cost, provide a tuneable stiffness, can operate over a broad range of temperatures and are simpler to process. However, some of the major drawbacks of SMPs are associated to its relatively low modulus, which results in a small recovery stresses/forces [10] (4-10 MPa against 200-400 MPa for SMA [11]), their response time [12] (greater than the tens of milliseconds for SMA) and their low achievable number of cycles.

Temperature has been the most used trigger, although there are alternative activation stimuli such as light [13,14], magnetic or electric fields [15-17], irradiation and $\mathrm{pH}$ changes [18]. In some cases, indirect heating is also achieved by inductive heating of SMP using magnetic [19] and electric fields, or irradiation.

Electro-active SMPs have been developed, mainly in what concerns its potential usage in actuators and artificial muscles. However, the relatively high response time (order of seconds) of the SMPs seems to constitute a drawback limiting its application span, especially for active and shape control. The latter seems also to draw the researcher's attention to the possibility of enhancing SMPs through polymeric matrices reinforcement. It is well established that the addition of nanoscale particles, such as carbon nanotubes (CNTs), into polymeric matrix holds the potential to improve the host material electrical, thermal and mechanical properties by orders of magnitude above the performance achieved by traditional fillers (such as glass fibers) [20]. The exceptional properties of the CNTs [21,22], due to its nanoscale diameter and high aspect ratio, have motivated an interest to exploit their properties in these SMPs, in order to improve the performance of the matrix polymer, i.e. the improve of its electrical conductivity as well as some of detrimental mechanical properties (i.e. elastic modulus) and an increase on its shape recovery stress [23].

The literature reports a few preliminary studies regarding shape-memory polyurethanes reinforced with CNTs, and the results reveal an enhancement in shape recovery stress, for $3.0 \mathrm{wt} \%$ of MWNT on shape memory polyurethanes fibers, suggesting a shorter recovery time [23]. These results highlight the enormous potential of this research line for distributed shape control, morphing mechanisms and other actuation devices, such as artificial muscles, opposing to the current solutions.

The main objective of this work is to evaluate the shape memory effect on the SMPU reinforced with CNTs prepared through two different techniques: in situ polymerization and by mechanical melt mixing. Before the dynamic characterization to evaluate the shape memory properties, some other experimental techniques must be performed in order to evaluate the final quality of the processed samples, such as the distribution of the CNTs into polymeric matrix, and evaluate its shape memory effect and its potential for the above mentioned systems. The effect on CNTs on thermal properties and on shape memory effect has been qualitatively evaluated.

\section{Experimental}

\subsection{Materials}

\subsubsection{For in situ polymerization}

The polycaprolactone diol was obtained from Perstorp CAPROLACTONES (CAPA ${ }^{\circledR}$ 2403D) with a molecular weight of 4000 and a typical $\mathrm{OH}$ value of $28 \mathrm{mg} \mathrm{KOH} / \mathrm{g}$. 4,4'-Methylenebis(phenyl isocyanate) (MDI) and 1,4-butanediol (BDO) were obtained from Aldrich with purity of 98\% and higher than 99\%, respectively. Dimethylformamide (DMF), triethylamine and glycerol were also used for the synthesis of the shape memory polyurethane.

\subsubsection{For mechanical melt mixing}

The thermoplastic polyurethane (TPU) was supplied by Lubrizol Corporation. The selected one was the Tecoflex TPU, which is a aliphatic polyether-based thermoplastic polyurethane. This TPU can be manufactured by extrusion and injection molding, and offer an inherent ultraviolet (UV) stability that resists to aging yellowing. The used Tecoflex TPU was the EG-60D-B20 with 20\% loading of barium sulfate. The typical data is listed in Appendix on Table A1. Due to the hygroscopic characteristics of the Thermedic Polymer Products, the pellets were dried prior to use, for $8 \mathrm{~h}$ in a furnace chamber, at $50^{\circ} \mathrm{C}$.

\subsubsection{Carbon nanotubes (CNTS)}

Two types of CNTs were used: (1) purchased from Shenzhen Nanotech PortCo., Lda with the following specifications: 20-40 $\mathrm{nm}$ diameter, $1-2 \mu \mathrm{m}$ length and a purity higher than 95\%; (2) purchased to CheapTubes, USA, and detain the following specifications: an outer diameter ranging from 50 to $80 \mathrm{~nm}$, an inner diameter ranging from 5 to $10 \mathrm{~nm}$, a length in a range of $10-20 \mu \mathrm{m}$, and a purity higher than $95 \mathrm{wt} \%$. As the pristine CNTs tend to agglomerate due to Van der Waals forces it is necessary to modify the CNTs in order to obtain a better dispersion within the polymeric matrixes. Chemical functionalization has shown to be an effective technique to improve the dispersion of carbon nanotubes $[24,25]$. In this work, the pristine CNT were functionalized via acid treatment to attach carboxylic groups to the surface [26]. A mixture of sulfuric and nitride acids were added to CNTs in a proportion of $(3: 1)$ and refluxed for $30 \mathrm{~min}$ at $140{ }^{\circ} \mathrm{C}$. Afterwards, the mixture is washed with deionised water (DW) until $\mathrm{pH} \sim 7$ and dried at $100^{\circ} \mathrm{C}$ in a furnace until evaporation of the DW. Fig. 1 shows the Fourier Transform Infrared (FT-IR) spectra for the functionalized and pristine CNTs. As it can be clearly observed, both samples exhibit a peak around $1630 \mathrm{~cm}^{-1}$, which represent the carbon skeleton $(\mathrm{C}=\mathrm{C})$. This peak shows that the integrity of the CNTs is not affected by the chemical treatment [27]. The peaks near 1164 and $1203 \mathrm{~cm}^{-1}$ are related to $\mathrm{C}-\mathrm{O}$ stretching of phenolic and carboxylic groups, and the peak at $1699 \mathrm{~cm}^{-1}$ corresponds to $\mathrm{C}=\mathrm{O}$ stretching of the same groups $[28,29]$. These results reveal a good quality of the functionalized CNTs.

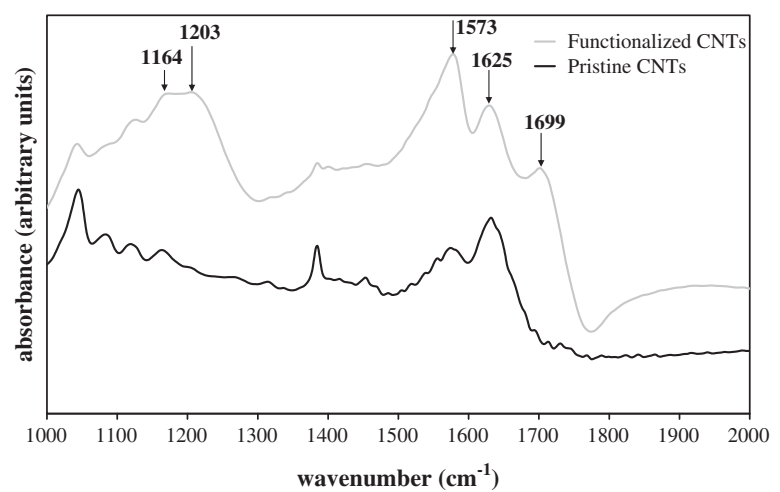

Fig. 1. FT-IR spectra for the functionalized and pristine CNTs. 


\subsection{In situ polymerization}

For the synthesis of the polyurethane (SMPU), the experimental setup shown in Fig. 2 was applied. A reactor, equipped with a mechanical stirrer, nitrogen inlet and condenser was used. First, $3 \mathrm{mmol}$ of PCL4000, $6 \mathrm{mmol}$ of MDI and $80 \mathrm{~mL}$ of DMF were charged into the reactor during a period of $2 \mathrm{~h}$ at $80^{\circ} \mathrm{C}$ and after, $2 \mathrm{mmol}$ of MDI were added, with the reaction being maintained at the same temperature. Then, $5 \mathrm{mmol}$ of BDO and $8 \mathrm{mmol}$ of MDI were added and the reaction continued for two additional hours.

After this period, the glycerol was charged into the reactor and $1 \mathrm{~h}$ later $2.2 \mathrm{mmol}$ of triethylamine were added slowly, at $50{ }^{\circ} \mathrm{C}$ for $30 \mathrm{~min}$. The polyurethane obtained was dried at $70-80{ }^{\circ} \mathrm{C}$ for $12 \mathrm{~h}$, resulting in translucent thin films. The addition of CNTs into the SMPU was performed during the polymerization of the PU, and in situ polymerization was therefore promoted. Nanocomposites with $0.1,0.25$ and $5.0 \%$ vol. CNTs were prepared.

The FT-IR spectrum for the in situ polymerization SMPU is shown in Fig. 3 and is compared with the spectra obtained for the nanocomposites: SMPU/0.1\%CNT; SMPU/0.25\%CNT and SMPU/0.50\%CNT. The SMPU spectrum shows a peak at $1725 \mathrm{~cm}^{-1}$ corresponding to the ester and urethane $\mathrm{C}=0$ stretching vibration and another two significant bands at 3310 and $1521 \mathrm{~cm}^{-1}$ due to $\mathrm{N}-\mathrm{H}$ vibration mode and another one at $2941 \mathrm{~cm}^{-1}$, related to

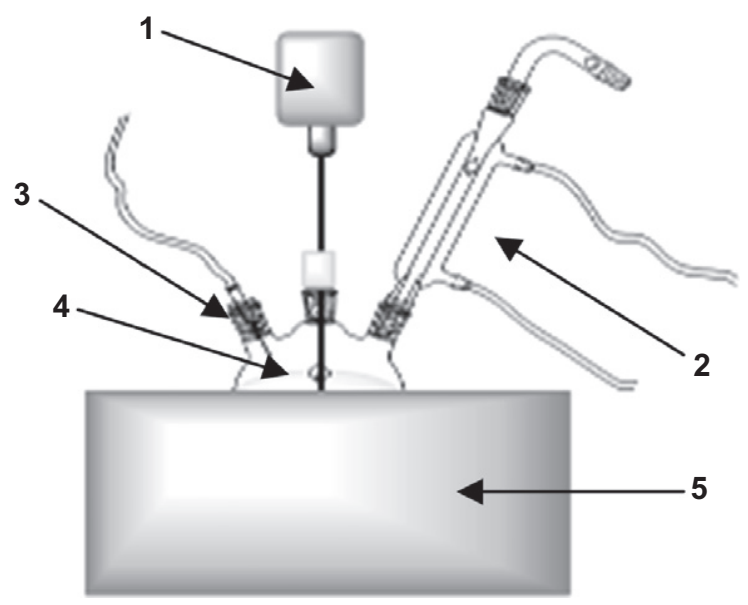

Fig. 2. Schematic representation of the experimental setup used for the synthesis of SMPUs and its nanocomposites (SMPU/CNT): (1) mechanical stirrer, (2) condenser, (3) nitrogen inlet, (4) reactor, and (5) thermostatic bath.

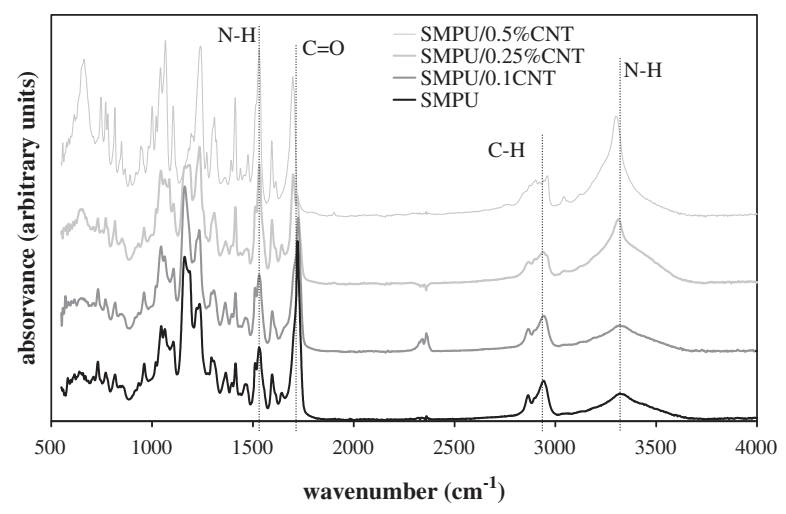

Fig. 3. FT-IR spectra of the final pure SMPU and of the synthesized nanocomposites: SMPU/0.1\%CNT, SMPU $/ 0.25 \%$ CNT and SMPU/0.50\%CNT. the $\mathrm{C}-\mathrm{H}$ vibration mode. The characteristic peaks of $\mathrm{OH}$ and $\mathrm{NCO}$ were absent, which means that $\mathrm{NCO}$ and $\mathrm{OH}$ groups reacted with the hydroxyl groups of PCL, leading to the formation of the polyurethane. The entire content of MDI was consumed, as well as the polycaprolactone. Furthermore, the spectra for different nanocomposites show similar peaks, though its intensity is slightly higher and the peak position moves toward lower wavenumber, from $1725 \mathrm{~cm}^{-1}$ to $1700 \mathrm{~cm}^{-1}$, with the increase of the CNT loading. This shift to lower wavenumber could be related to an increase of the interaction of the host polymer and the nanoparticles $[28,30]$.

\subsection{Mechanical melt mixing}

The incorporation of the CNTs into raw TPU was performed by melt processing with an Internal Batch Mixer. The used temperature of mixture was $185^{\circ} \mathrm{C}$, with a velocity of $100 \mathrm{rpm}$ and for $10 \mathrm{~min}$. After mixing, the new composites are processed through injection molding in a micro-injection machine, with the following injection conditions: $T_{\text {injection }}=190^{\circ} \mathrm{C}, \quad T_{\text {mold }}=90^{\circ} \mathrm{C}, \quad P_{\text {injection }}=$ $700 \mathrm{bar}, P_{\text {compaction }}=750 \mathrm{bar}, t_{\text {injection }}=20 \mathrm{~s}$ and $t_{\text {compaction }}=25 \mathrm{~s}$. The obtained samples are dumb bell shaped with the following dimensions: $2 \mathrm{~mm}$ thick, $4 \mathrm{~mm}$ neck width and $22 \mathrm{~mm}$ neck long.

\subsection{Instrumental analysis}

Standard methods of characterization, such as Scanning Electron Microscopy (SEM), Fourier Transform Infra-red (FT-IR), Thermogravimetricl Analysis (TGA), Differential Scanning Calorimetry (DSC) and Dynamic Mechanical Thermal Analysis (DMA) were used in this study with the purpose of analyzing and characterize the obtained samples (in situ polymerization SMPU/CNT and mechanical melt mixing TPU/CNT nanocomposites).

The samples morphology was evaluated by a Hitachi SU-70 SEM, with a resolution of $1 \mathrm{~nm}$ at $15 \mathrm{KV}$. The functionalized CNTs were characterized by FT-IR analysis in a Brucker Tensor-27 spectrometer in the range of $400-4000 \mathrm{~cm}^{-1}$. A JASCO FT-IR 4100 equipment was used to characterize the synthesized nanocomposites. The thermal stability of the polyurethane was evaluated by using a TA Instruments Q500 thermogravimetric analyzer (thermobalance sensitivity: $0.1 \mu \mathrm{g}$ ). The temperature calibration was carried out in the range $25-1000{ }^{\circ} \mathrm{C}$ by measuring the Curie point of nickel standard, using open platinum crucibles. Dry nitrogen purge flow of $100 \mathrm{~mL} / \mathrm{min}$ and a heating rate of $10^{\circ} \mathrm{C} / \mathrm{min}$, summarize the conditions applied for the thermoanalytical measurements. The quantity analyzed in each sample did not exceed the $17 \mathrm{mg}$. The DSC studies were performed in a TA Q100 instrument with a heating rate of $10{ }^{\circ} \mathrm{C} \mathrm{min}^{-1}$ and with a nitrogen flux of $50 \mathrm{~mL} \mathrm{~min}^{-1}$. Fusion temperatures $\left(T_{f}\right)$ where determined using this technique. DMTA analyses were performed in a Tritec 2000 DMA equipment with a heating rate of $5{ }^{\circ} \mathrm{C} \mathrm{min}{ }^{-1}$, in the range of temperatures from $-150^{\circ} \mathrm{C}$ to $250^{\circ} \mathrm{C}$. By thermal analyses it was possible to determine glass transition temperature $\left(T_{g}\right)$.

\section{Results and discussion}

As already mentioned, FT-IR was employed to analyze the quality of the functionalized CNTs. The induced carboxylic groups on the CNTs are expected to enhance the interaction between CNTs and TPU. Both types of samples (in situ polymerization SMPU/ CNT and mechanical melt mixing TPU/CNT nanocomposites) were characterized through Scanning Electron Microscopy (SEM), before and after processing by injection molding. Fig. 4 shows some pictures of the different samples. As it can be observed, for both types of samples, the depicted pictures show that CNTs are completely 


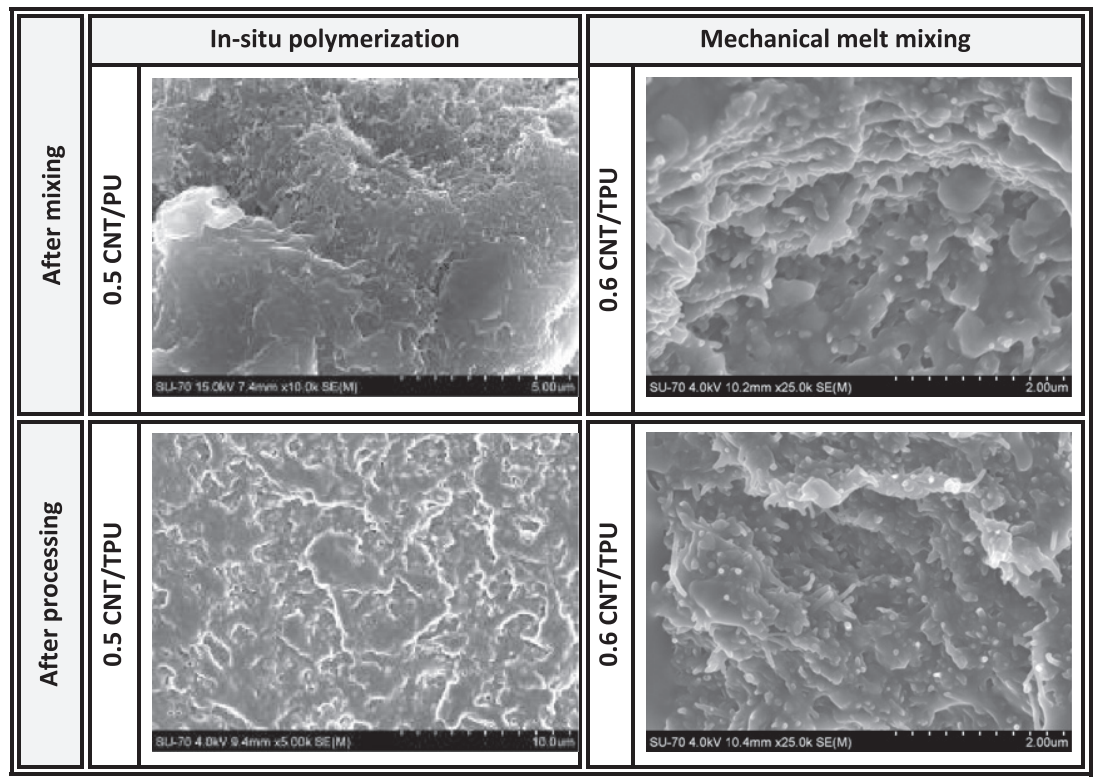

Fig. 4. SEM pictures from the nanocomposites prepared through in situ polymerization and mechanical melt mixing, before and after processing by injection molding.
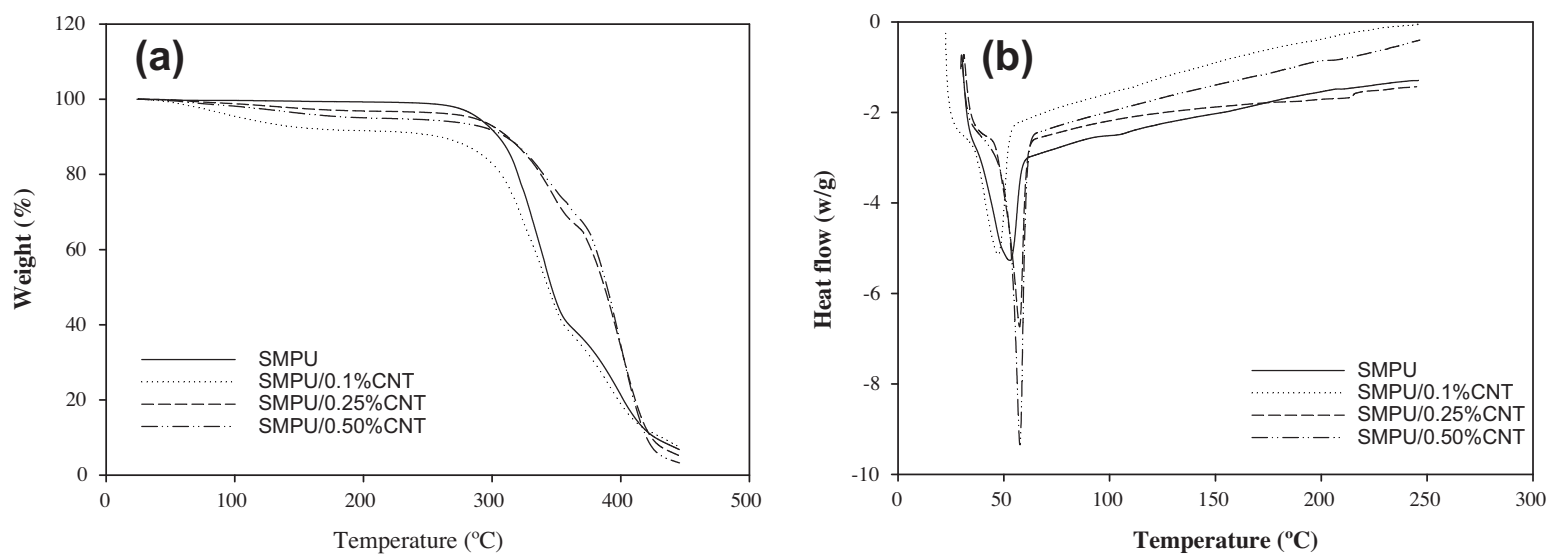

Fig. 5. (a) TGA and (b) DSC measurements for the synthesized pure SMPU and their nanocomposites.

immersed into the polymeric matrix. After processing through injection molding, the samples prepared by in situ polymerization show that CNTs are well dispersed, as well for the mechanical melt mixing samples. These SEM pictures clearly show that the CNTs are well incorporated into TPU matrix, and that they keep their integrity, for the both types of studied samples. Also it is possible to verify that there is no evidence for CNT aggregation or agglomeration. The inexistence of these effects is due to the chemical treatment previously performed to CNTs, which facilitate the higher degree of wetting by the SMPU and phase adhesion between them [31]. It is well known that the existence of aggregates or agglomerates could cause rupture points causing the deterioration of the mechanical and thermal properties of the composites. The quite good homogeneity of the CNTs into SMPU provides a way to explain the enhancement of composites properties. Although the morphological results are identical for both types of samples, it can be claimed that the mechanical melt mixing is a quite simpler and efficient method to obtain a homogeneous nanocomposite, with enhanced properties, detaining promising features to a possible industrial implementation of this technique to produce homogeneous nanocomposites.

The thermal stability of the in situ polymerization SMPU and its nanocomposites (with $0.1,0.25$ and $0.5 \%$ vol CNTs) was evaluated by Thermogravimetric analysis (TGA) and differential scanning calorimetry (DSC). The spectra of TGA presented in Fig. 5a shows that, for the pure SMPU, a first step can be observed for $315.80{ }^{\circ} \mathrm{C}$, which is believed to be due to the evaporation of the solvent (dimethylformamide) in the PU, corresponding to the degradation of the polymer (degradation temperature $-T_{d}$ ). With increasing CNT loading (from 0.1 to $0.50 \%$ vol CNTs) it can be observed that the degradation temperature increases. In Fig. 5b the DSC curves can be observed. The glass transition temperatures could not be calculated from these curves since the range temperatures of the DSC equipment was limited to the range between $25^{\circ} \mathrm{C}$ and $500{ }^{\circ} \mathrm{C}$. Nevertheless, the fusion temperature $\left(T_{f}\right)$ was determined. The $T_{f}$ obtained from DSC curves were: $52.8^{\circ} \mathrm{C}, 47.30^{\circ} \mathrm{C}, 57.10^{\circ} \mathrm{C}$ and $57.80{ }^{\circ} \mathrm{C}$ for pure SMPU, SMPU/0.1\%CNT, SMPU/0.25\%CNT and SMPU $/ 0.50 \% C N T$, respectively. As it can be observed, the fusion temperature increases as the concentration of CNTs increases.

Dynamic mechanical thermal analysis (DMTA) was performed to validate those measurements obtained by TGA and DSC analysis, and to determine the glass transition temperature $\left(T_{g}\right)$ of the in situ polymerization samples. Fig. 6 shows the spectra for pure SMPU and its nanocomposites. The main differences in DMTA spectra are observed in $\tan \delta$ value (also called loss factor and indicates damping). The incorporation of the CNTs increases the $\tan \delta$ value. 


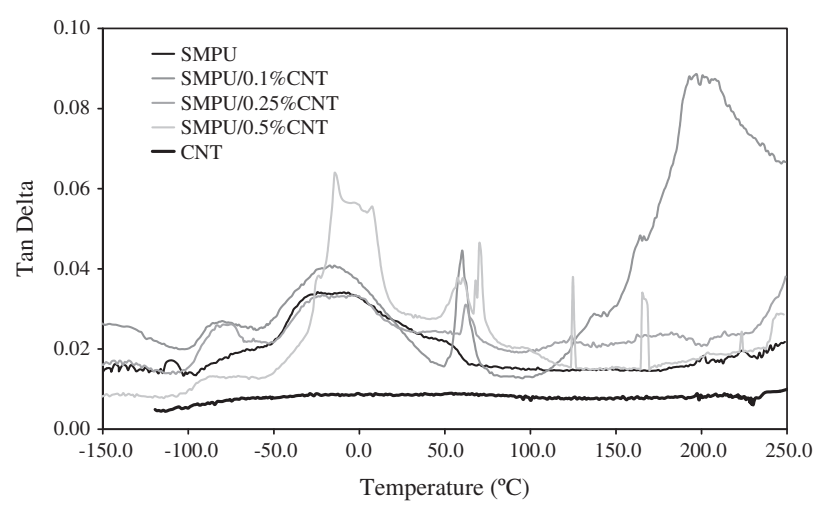

Fig. 6. DMTA spectra for the synthesized samples. The peaks for the glass transition temperature $\left(T_{g}\right)$ and the fusion temperature $\left(T_{f}\right)$ are identified.

This value is related with the dissipated energy in the material, i.e., gives the capacity of a material to absorb energy and dissipate it as heat, which depends on the temperature, the material state and the frequency. It is also shown that with increasing CNT concentration the glass transition temperature rises as well as the fusion temperature, an observation in agreement with the results obtained through DSC technique. Table compares the observed temperatures for each thermal technique (for the in situ polymerization samples). As it can be observed from Table 1 , the determined $T_{f}$ by DSC and complemented with DMTA technique has a maximum difference of $11^{\circ} \mathrm{C}$ for the nanocomposites with $0.1 \% \mathrm{CNT}$, which may not be significant. However, for both techniques (DSC and DMTA) it can be observed a significant enhancement on $T_{f}$ as CNT concentration increases. The same effect was observed for $T_{d}$ and $T_{g}$ temperatures. The SMPU/0.1\%CNT samples show a decrease in $T_{f}$, which may be related with the heterogeneity of the CNTs into the polymeric matrix. All the other samples presented an improvement on their thermal stability and mechanical properties. A more suitable determination of $T_{f}$ is crucial for the shape memory effect characterization.

The thermal characterization of the mechanical melt mixing samples was performed by DMTA. Fig. 7 presents the storage modulus $\left(E^{\prime}\right)$ and the $\tan \delta$ curves for the pure TPU and TPU/0.6\%vol. CNT nanocomposite, as function of temperature. The plot of the storage modulus $\left(E^{\prime}\right)$ versus temperature shows an increase of $E^{\prime}$ with the addition of CNTs (Fig. 7a), which may be attributed to the stiffness effect of CNTs [32]. The glass transition temperature, for the soft segment of TPU, is defined as the peak of the $\tan \delta$ curve, which is $43{ }^{\circ} \mathrm{C}$ and $50{ }^{\circ} \mathrm{C}$ for the TPU and TPU/0.6\%CNT, respectively. The observed transitions are common to polymers that exhibit useful shape-memory response [33]. The curves clearly show an increasing of the transition temperatures with increasing CNT loading (from $43^{\circ} \mathrm{C}$ to $50{ }^{\circ} \mathrm{C}$ ), which could be related to the good distribution of the CNTs into polymeric matrix, as discussed earlier, as well as due to the possible formation of rigid structure through different molecular interaction [30,34,35]. The peak high decreases for the nanocomposite which may be related to an improvement of the crosslinking density with the addition of CNTs, resulting in a significant decreasing on damping capacity of the TPU [36]. It is expected that this rise of the transition temperature, for the nanocomposite as compared with pure polyurethane, will be reflected in an enhancement of the shape recovery value of the nanocomposites [30].

As it is well known, it is expected that the inclusion of carbon nanotubes in a polymeric matrix improves the overall properties of the polymeric matrix. In this work it was shown that the incorporation of CNTs into polyurethane increases its thermal stability (with increasing degradation temperature - Table 1) and it was also observed an increasing on $T_{g}$, with the addition of CNTs,

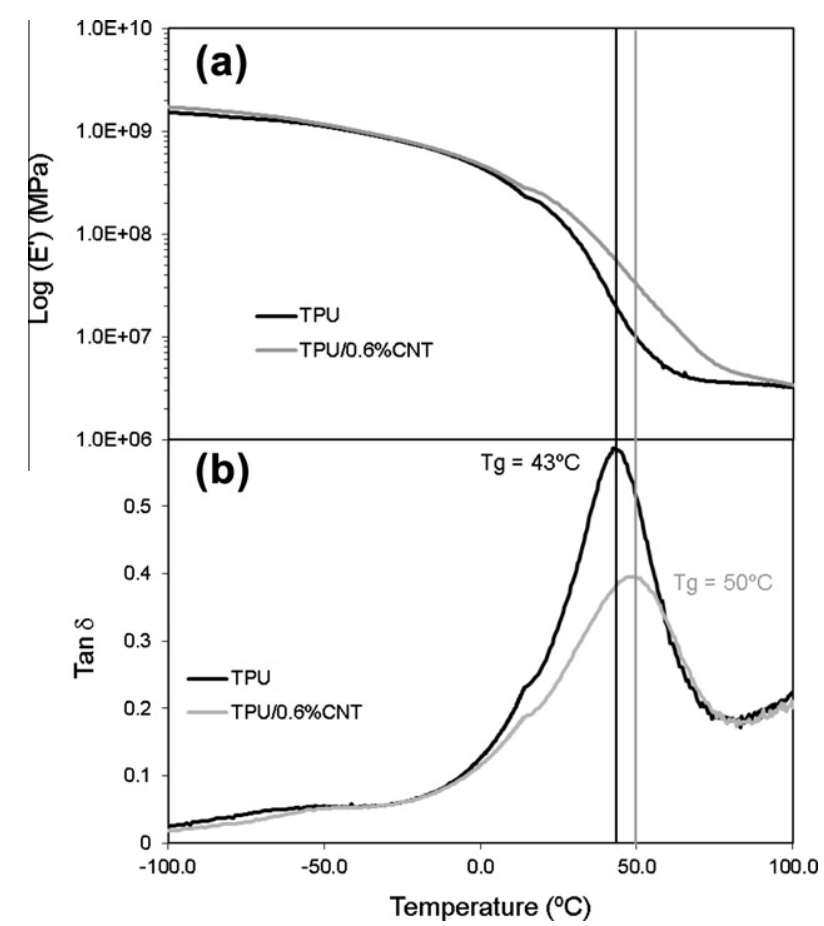

Fig. 7. (a) Storage Modulus (E') and (b) $\tan \delta$ spectra of the pure TPU and TPU/ $0.6 \% \mathrm{CNT}$ nanocomposite, as function of temperatures. The glass transition temperatures are defined by the $\tan \delta$ peak and are $43^{\circ} \mathrm{C}$ and $50{ }^{\circ} \mathrm{C}$ for the pure TPU and TPU/0.6\%CNT, respectively.

Table 1

Thermal properties of the synthesized SMPU analyzed by TGA, DSC and DMTA.

\begin{tabular}{lllll}
\hline Sample & $T_{g}\left({ }^{\circ} \mathrm{C}\right)$ & & $T_{f}\left({ }^{\circ} \mathrm{C}\right)$ & \\
\cline { 2 - 3 } & DMTA & DSC & DMTA & TGA \\
\hline SMPU & -25 & 52.8 & 49 & 307.5 \\
SMPU/0.1\%CNT & -22 & 47.3 & 58 & 313.4 \\
SMPU/0.25\%CNT & -20 & 57.1 & 61.9 & 340 \\
SMPU/0.5\%CNT & -16 & 57.8 & 68 & 360 \\
\hline
\end{tabular}

denoting an homogeneous distribution of the CNTs into the polyurethane matrix. To evaluate the effect of the addition of CNTs into TPU on mechanical properties of the processed nanocomposite, some tensile tests were carried out using a Shimadzu Universal Testing Machine model AG-50kNG (Shimadzu, Kyoto, Japan) with a video extensometer. The experiments were conducted at room temperature at a cross head speed of $50 \mathrm{~mm} / \mathrm{min}$ The results have shown that the addition of CNTs affects the mechanical properties of pure TPU. However for the used concentrations of CNTs ( 0.4 and $0.6 \% \mathrm{vol}$ ) the differences are not too significant. As the final goal of this work is to evaluate the shape memory properties of the pure TPU and its nanocomposites, the mechanical tests were made just to verify if the introduction of CNTs do in fact modify the mechanical properties of the TPU.

The shape-memory effect of both types of samples was tested through some preliminary experiments to evaluate this effect. To accomplish that, a furnace chamber with controlled temperature and humidity was used. The cycle applied to the samples is showed in Fig. 8 and can be described as follows: (1) The samples were heated from room temperature (RT) to $T_{\text {trans }}+20^{\circ} \mathrm{C}$; (2) keep at $T_{\text {trans }}+20^{\circ} \mathrm{C}$ for $10 \mathrm{~min}$; (3) An arbitrary strain was applied to deform the sample, and kept for $10 \mathrm{~min}$, at $T_{\text {trans }}+20^{\circ} \mathrm{C}$; (4) The samples were cooled to $T_{\text {trans }}-20^{\circ} \mathrm{C}$, with the applied strain, and kept for $10 \mathrm{~min}$; (5) The imposed strain was removed. At the end of this cycle the samples keep the intended shape. To recover the original shape, the samples were heated to $T_{\text {trans }}+20^{\circ} \mathrm{C}$, recovering its 


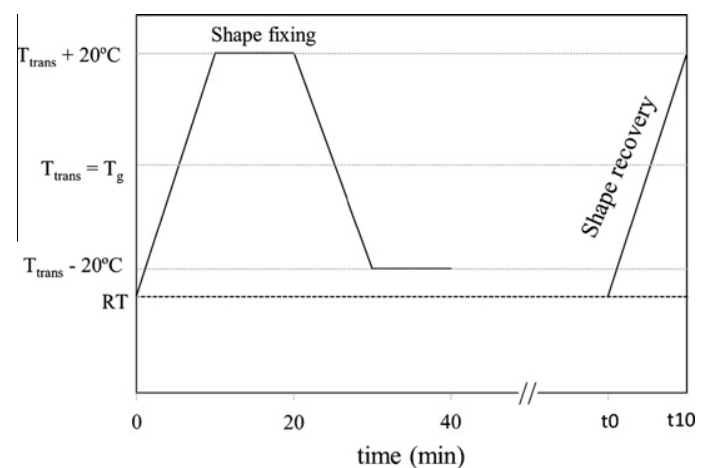

Fig. 8. Shape memory cycle applied to the mechanical melt mixing samples (pure TPU and its nanocomposites).

original shape without any residual deformation. It was observed that both types of samples exhibit shape memory properties. However, as it was already discussed, it is believed that the mechanical melt mixing nanocomposites can be easily processed in a large scale, as compared with the in situ polymerized nanocomposites which only can be processed in a laboratorial scale, with similar results. For that reason, all the dynamic characterization, to evaluate qualitatively the shape memory effect, was performed only to the mechanical melt mixing nanocomposites.

An experimental setup was designed and implemented to evaluate the shape memory effect of the processed samples. In Fig. 9 a sequential set of photographs is represented, showing the shape recovery for the pristine TPU sample as well as for the nanocomposites samples (TPU/0.4\%CNT and TPU/0.6\%CNT). As observed, all studied samples show shape memory properties, and these preliminary tests reveal that the recovery time of the nanocomposite samples was smaller than that observed for the pure TPU, The decreasing of recovery time with increasing CNT volume fraction is in accordance with the observations by Meng and $\mathrm{Hu}$ [23]. These results reveal that the CNTs act as nucleating agents, and hence improve the crystallinity of the soft segments, as well as the mechanical properties of the TPU.

Though it was shown that the processed samples (pure TPU as well as the nanocomposites) detain shape-memory effect it is mandatory to develop and monitor further each step of the described process, in order to determine and quantify the shape recovery, the shape setting and recovery time of the TPU and its CNTs nanocomposites. It is of utmost importance to evaluate the effects of the CNTs into holding capacity and recovery time, and prove the results obtained through DMTA, in which the increase in storage modulus and the increase of the glass transition temperature

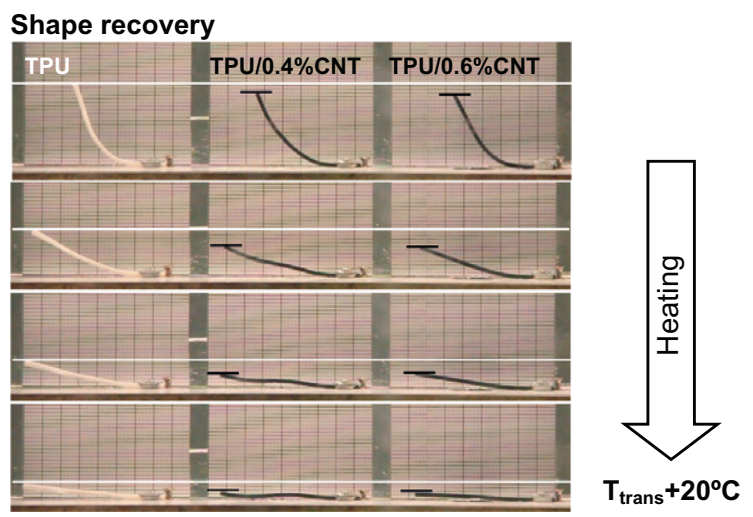

Fig. 9. Sequential set of photographs showing the shape recovery of the processed samples: pristine TPU and nanocomposites (TPU/0.4\%CNT and TPU/0.6\%CNT). may be related with an increase of the shape recovery ratio, and, hence return to its original shape faster than pristine TPU.

\section{Conclusions}

In this paper, samples processed through two different processing techniques, namely in situ polymerization SMPU and mechanical melt mixing, were processed and analyzed. The chemically treated CNTs were mixed into polyurethane, and the FTIR analysis has shown that the surface of the CNTs was chemically activated through the introduction of carboxylic groups, which facilitates the bonding with the polyurethane matrix. The SEM analysis shows that the CNTs were well incorporated into polyurethane matrix and that there is no evidence of agglomeration and/or aggregation of CNTs, for both types of samples. These results denote that the mechanical melt mixing technique is able to obtain good quality samples, rending promising features to a possible industrialization of these tailored nanocomposites. The thermal analysis has shown that the degradation temperature of the SMPU occurs between 290 and $400{ }^{\circ} \mathrm{C}$. The weight loss in the first region can be related with the degradation of the hard segment while the second region can be due to degradation of the soft segment. The incorporation of CNTs into the polymer matrix resulted in an increase of $T_{f}, T_{d}$ and $T_{g}$ for all samples. This increase could be related to the good distribution of CNTs which can justify the shape recovery values of the nanocomposites, as well as the decreasing on time recovery, as it was observed. The expeditious dynamic tests to assess the shape memory effect highlight the initially predicted potential. In fact a decreasing of the recovery time for the nanocomposite was observed in comparison to the pure polyurethane response, the latter stressing the need for further insight and improvement to this specific test rig, as well as the required testing methodology for results accuracy.

\subsection{Future work}

Evaluate, and quantify the shape memory properties of the prepared samples (with a parametric study of different volume fractions of CNTs), and determine the shape recovery and shape holding properties.

\section{Acknowledgments}

Authors acknowledge to Fundação para a Ciência e Tecnologia (FCT) for its funding support through the Project TDC/EME-TME/ 104178/2008 and through the individual Grant SFRH/BPD/81791/ 2011.

\section{Appendix A}

Table A1.

Table A1

Typical Physical Data for EG-60D-B20.

\begin{tabular}{lll}
\hline Product & & \\
\hline Property & ASTM test & EG-60D-B20 \\
\hline Durometer (shore hardness) & D2240 & $55 \mathrm{D}$ \\
Specific gravity & D792 & 1.32 \\
Flexural modulus (psi) & D790 & 27000 \\
Ultimate tensile (psi) & D412 & 7500 \\
Ultimate elongation (psi) & D412 & 370 \\
Tensile (psi) & D412 & \\
At 100\% elongation & & 2000 \\
At 200\% elongation & & 3100 \\
At 300\% elongation & & 6000 \\
Mold shrinkage (in/in) & & $0.04-0.08$ \\
\hline
\end{tabular}




\section{References}

[1] Friswell MI. The prospects for morphing aircraft. IV ECCOMAS-SMART'09. Porto, Portugal; 2009.

[2] Paradies R, Hertwig M. Shape control of adaptive composite reflectors. Compos Part B: Eng 1999;30:65-78.

[3] Irschik H. A review on static and dynamic shape control of structures by piezoelectric actuation. Eng Struct 2002;24:5-11.

[4] Ma K, Ghasemi-Nejhad MN. Adaptive precision positioning of smart composite panels subjected to external disturbances. Mechatronics 2006; 16:623-30.

[5] Preumont A. Vibration control of active structures: an Introduction: Springer Netherlands; 2011.

[6] Gardonio P. Sensors and actuators for active structural acoustic and active vibration feedback control. In: Rodrigues ACaJD, editor. IV ECCOMASSMART'09. Porto-Portugal2009. p. 215-34.

[7] Liu S, Tong L, Lin Z. Simultaneous optimization of control parameters and configurations of PZT actuators for morphing structural shapes. Finite Elements Anal Design 2008;44:417-24.

[8] James T, Menner A, Bismarcj A, Iannucci L. Morphing skins: development of new hybrid materials. 4th SEAS DTC. Edinburgo; 2009.

[9] Hugh AB, Charles LM, Theresa LV. Repeatable bending actuation in polyurethanes using opposing embedded one-way shape memory alloy wires exhibiting large deformation recovery. Smart Mater Struct 2002;11:509.

[10] Koerner H, Price G, Pearce NA, Alexander M, Vaia RA. Remotely actuated polymer nanocomposites[mdash]stress-recovery of carbon-nanotube-filled thermoplastic elastomers. Nat Mater 2004;3:115-20.

[11] Buckley CP, Prisacariu C, Caraculacu A. Novel triol-crosslinked polyurethanes and their thermorheological characterization as shape-memory materials. Polymer 2007; 48:1388-96.

[12] Xu J, Shi W, Pang W. Synthesis and shape memory effects of Si-O-Si crosslinked hybrid polyurethanes. Polymer 2006;47:457-65.

[13] Lendlein A, Jiang H, Junger O, Langer R. Light-induced shape-memory polymers. Nature 2005;434:879-82.

[14] Jiang HY, Kelch S, Lendlein A. Polymers move in response to light. Adv Mater 2006;18:1471-5.

[15] Mirfakhrai T, Madden JDW, Baughman RH. Polymer artificial muscles. Mater Today 2007:10:30-8.

[16] Lu TJ, Evans AG. Design of a high authority flexural actuator using an electrostrictive polymer. Sensors Actuators A: Phys 2002;99:290-6.

[17] Liu Y, Lv H, Lan X, Leng J, Du S. Review of electro-active shape-memory polymer composite. Compos Sci Technol 2009;69:2064-8.

[18] Harris KD, Bastiaansen CWM, Broer DJ. Physical properties of anisotropically swelling hydrogen-bonded liquid crystal polymer actuators. J Microelectromech Syst 2007;16:480-8.

[19] Buckley PR, McKinley GH, Wilson TS, Small W, Benett WJ, Bearinger JP, et al. Inductively heated shape memory polymer for the magnetic actuation of medical devices. IEEE Trans Biomed Eng 2006;53:2075-83.
[20] Paul DR, Robeson LM. Polymer nanotechnology: nanocomposites. Polymer 2008;49:3187-204.

[21] Ebbesen TW. Carbon nanotubes. Phys Today 1996;49:26-32.

[22] Coleman JN, Khan U, Blau WJ, Gun'ko YK. Small but strong: a review of the mechanical properties of carbon nanotube-polymer composites. Carbon 2006;44:1624-52.

[23] Meng Q, Hu J. Self-organizing alignment of carbon nanotube in shape memory segmented fiber prepared by in situ polymerization and melt spinning. Compos Part A: Appl Sci Manuf 2008;39:314-21.

[24] Jung YC, Sahoo NG, Cho JW. Polymeric nanocomposites of polyurethane block copolymers and functionalized multi-walled carbon nanotubes as crosslinkers. Macromol Rapid Commun 2006;27:126-31.

[25] Kwon J, Kim H. Comparison of the properties of waterborne polyurethane/ multiwalled carbon nanotube and acid-treated multiwalled carbon nanotube composites prepared by in situ polymerization. J Polym Sci Part A: Polym Chem 2005;43:3973-85.

[26] Esumi K, Ishigami M, Nakajima A, Sawada K, Honda H. Chemical treatment of carbon nanotubes. Carbon 1996;34:279-81.

[27] Rahimpour A, Jahanshahi M, Khalili S, Mollahosseini A, Zirepour A, Rajaeian B. Novel functionalized carbon nanotubes for improving the surface properties and performance of polyethersulfone (PES) membrane. Desalination 2012;286:99-107.

[28] Kim JY, Han SI, Hong S. Effect of modified carbon nanotube on the properties of aromatic polyester nanocomposites. Polymer 2008;49:3335-45.

[29] Zhang J, Zou H, Qing Q, Yang Y, Li Q, Liu Z, et al. Effect of chemical oxidation on the structure of single-walled carbon nanotubes. J Phys Chem B 2003;107: 3712-8.

[30] Deka H, Karak N, Kalita RD, Buragohain AK. Biocompatible hyperbranched polyurethane/multi-walled carbon nanotube composites as shape memory materials. Carbon 2010;48:2013-22.

[31] Chen W, Tao X, Liu Y. Carbon nanotube-reinforced polyurethane composite fibers. Compos Sci Technol 2006;66:3029-34

[32] Jin Z, Pramoda KP, Xu G, Goh SH. Dynamic mechanical behavior of meltprocessed multi-walled carbon nanotube/poly(methyl methacrylate) composites. Chem Phys Lett 2001;337:43-7.

[33] Liu C, Chun SB, Mather PT, Zheng L, Haley EH, Coughlin EB. Chemically crosslinked polycyclooctene: synthesis, characterization, and shape memory behavior. Macromolecules 2002;35:9868-74.

[34] Sahoo NG, Jung YC, Yoo HJ, Cho JW. Influence of carbon nanotubes and polypyrrole on the thermal, mechanical and electroactive shape-memory properties of polyurethane nanocomposites. Compos Sci Technol 2007;67: 1920-9.

[35] Sravendra R, Niranjan K, Jae Whan C, Young Ho K. Enhanced dispersion of carbon nanotubes in hyperbranched polyurethane and properties of nanocomposites. Nanotechnology 2008;19:495707.

[36] Barick AK, Tripathy DK. Effect of nanofiber on material properties of vaporgrown carbon nanofiber reinforced thermoplastic polyurethane (TPU/CNF) nanocomposites prepared by melt compounding. Compos Part A: Appl Sci Manuf 2010;41:1471-82. 